\title{
Human Papillomavirus-16 DNA Quantitation Differentiates High-Grade Anal Neoplasia
}

\author{
Melissa Agsalda-Garcia ${ }^{1,2}$, Tiffany Shieh ${ }^{1,2}$, Eleanore Chuang ${ }^{1,2}$, Nicholas Loi ${ }^{1,2}$, Cris Milne ${ }^{1}$, \\ Rui Fang ${ }^{3}$, Eunjung Lim ${ }^{3}$, Jeffrey Killeen ${ }^{4,5}$ and Bruce Shiramizu 1,2,* (i) \\ 1 Hawaii Center for AIDS, Honolulu, HI 96813, USA; magsalda@hawaii.edu (M.A.-G.); \\ tshieh@hawaii.edu (T.S.); eleanore@hawaii.edu (E.C.); NLoi@hawaii.edu (N.L.); cmilne@hawaii.edu (C.M.) \\ 2 Department of Tropical Medicine, Medical Microbiology, and Pharmacology, John A. Burns School of \\ Medicine, University of Hawaii, Honolulu, HI 96813, USA \\ 3 Complementary and Integrative Medicine, John A. Burns School of Medicine, University of Hawaii, \\ Honolulu, HI 96813, USA; fangr@hawaii.edu (R.F.); lime@hawaii.edu (E.L.) \\ 4 University of Hawaii Cancer Center, Honolulu, HI 96813, USA; Jeffrey.Killeen@hawaiilabs.com \\ 5 Pathology Department, John A. Burns School of Medicine, University of Hawaii, Honolulu, HI 96813, USA \\ * Correspondence: bshirami@hawaii.edu; Tel.: +1-808-692-1677
}

Received: 16 July 2018; Accepted: 4 August 2018; Published: 8 August 2018

\begin{abstract}
Background: Due to their higher rates of anal dysplasia/cancer, human immunodeficiency virus (HIV)-positive individuals are recommended to undergo anal dysplasia screening, which consists of anal cytology (AC) and high resolution anoscopy (HRA) with anal biopsy (AB) after abnormal AC result. However, AC variability limits its usefulness. Our objective was to evaluate human papillomavirus (HPV)-16 DNA quantitation as part of the screening algorithm. Methods: HPV-16 was detected in AC specimens from 75 HIV-positive participants using quantitative real-time polymerase chain reaction. $\mathrm{AB}$ results were available from 18/44 patients who had abnormal AC. Statistical tests included Mann-Whitney U, Kruskal-Wallis, receiver operating characteristic (ROC) analysis and Kappa coefficient tests. Results: HPV-16 copy numbers differed significantly across AC $(p=0.001)$ and $\mathrm{AB}$ grades $(p=0.009)$. HPV-16 $\geq 65$ copies/cell predicted high-grade $\mathrm{AB}(p=0.04)$. Using this cut-off in comparison to $\mathrm{AB}$, it had better specificity (1.00) than $\mathrm{AC}(0.75)$ and specificity (0.77) than qualitative HPV-16 detection (0.38). Also, the Kappa coefficient of the cut-off $(\mathrm{K}=0.649)$ was higher than AC $(K=0.557)$ and qualitative HPV-16 detection $(K=0.258)$ to AB. Conclusion: Higher HPV-16 copy numbers corresponded to higher AC and AB grades, suggesting the importance of HPV burden on disease stage. Furthermore, HPV-16 $\geq 65$ copies/cell distinguished high-grade disease and demonstrated better sensitivity, specificity, and agreement with AB than AC or qualitative HPV-16 detection. These results support the potential use of HPV quantitation in conjunction with AC in anal dysplasia screening.
\end{abstract}

Keywords: human papillomavirus; HPV; HIV/AIDS; men who have sex with men; MSM; anal cancer; cytology; dysplasia

\section{Introduction}

Human immunodeficiency virus (HIV)-infected individuals are at a higher risk for developing anal dysplasia and cancer, particularly men who have sex with men (MSM) [1-4]. Histopathological changes in the squamous epithelium of the transitional zone manifest from human papillomavirus (HPV) infection and lead to anal squamous intraepithelial lesions (ASIL) ranging from low-grade to high-grade SIL (LSIL, HSIL) which represents the spectrum of anal dysplasia pathologies including those associated with high-risk HPV types, with HPV-16 accounting for the majority of anal cancer 
cases [2]. Due to their increased risk for anal dysplasia/cancer, HIV-infected individuals are recommended to undergo regular anal cytology (AC) screening [5]. AC grades are classified as atypical squamous cells of undetermined significance (ASCUS), atypical squamous cells but cannot exclude high-grade (ASC-H), low-grade squamous intraepithelial lesions (LSIL), or high-grade squamous intraepithelial lesions (HSIL). If an individual is found to have an abnormal AC, he/she is recommended to undergo more invasive high resolution anoscopy (HRA), the current gold standard in ASIL and anal cancer diagnosis. During HRA, biopsies are taken from areas with apparent morphological changes in tissue. Abnormal anal biopsy (AB) are classified using the lower anogenital squamous terminology (LAST) [6] as either low-grade squamous intraepithelial lesions (LSIL) or high-grade squamous intraepithelial lesions (HSIL).

Currently, treatment is recommended only for those with high-grade disease. Because low-grade disease is not typically treated, screening that identifies LSIL prior to HRA and AB could reduce the number of invasive procedures that appear unnecessary in retrospect. Another factor that influences $\mathrm{AC}$ results is its inherent sensitivity and specificity as a screening tool [7]. Although AB during HRA is considered the current gold standard, it is also subject to variability in sensitivity and specificity, which highlights the need for improved screening with consistent sensitivity and specificity for high-grade lesions to prevent unnecessary HRAs, ABs, and other interventions [7-10].

Since HPV-16 is the most prevalent high-risk HPV genotype in anal dysplasia/cancer and is associated with high-grade lesions [11,12], the objective of the study was to determine if HPV-16 DNA quantitation could improve the screening algorithm in distinguishing low-grade disease from high-grade disease. Ultimately, results of the study could decrease the number of HRAs and ABs performed for low-grade disease. We hypothesized that higher HPV-16 DNA copy numbers would correspond to high-grade AC and AB. In this study, we found that HPV-16 DNA copy numbers differed according to $\mathrm{AC}$ and $\mathrm{AB}$ grades and that a cut-off value of $65 \mathrm{HPV}-16$ copies per cell, as determined by receiver operating characteristics (ROC) analysis, differentiated low-grade from high-grade disease and provided high sensitivity and specificity. These findings support the utility of adding HPV-16 quantitation to the anal dysplasia screening algorithm.

\section{Materials and Methods}

\subsection{Patient Enrollment and Specimen Collection}

For the Hawaii Center for AIDS RMATRIX Pilot Project RM004, 75 HIV-positive patients enrolled and provided consent under a protocol approved by the University of Hawaii Institutional Review Board (CHS \#21953). AC specimens were collected in ThinPrep Collection vials during one study visit for routine healthcare maintenance and high-risk HPV (hr-HPV) assessment. Abnormal AC results were reported as ASCUS, ASC-H, LSIL, or HSIL. Participants were followed up after the AC results were available as part of routine medical care, and HRA was recommended to those with abnormal AC. If HRA was performed, and biopsies were obtained, biopsy results from participants who gave consent were available for the study. AB pathologies were reported as normal, LSIL, or HSIL.

\subsection{Anal hr-HPV}

DNA was extracted from AC specimens, using the Machery-Nagel NucleoSpin Tissue XS Kit, and quantitated on a NanoDrop 2000 instrument. Specimens were analyzed for HPV-16 genotype by quantitative real-time PCR (qRT-PCR), using primers and probes targeting the E6/E7 oncogenic region [13] and $\beta$-globin for the housekeeping gene. Analyses were completed using SDS 2.3 software (Applied Biosystems, Foster City, CA, USA). Standard curves were derived from ten-fold serial dilutions of $\beta$-globin plasmid and HPV-16 plasmid-p1203 PML2d HPV-16 was a gift from Peter Howley (AddGene \#10869)—at calculated quantities from 10 copies to 1 million copies. Controls included DNA from SiHa cells (American Type Culture Collection ATCC ${ }^{\circledR}$ HTB-35 ${ }^{\mathrm{TM}}$ ), positive for HPV-16; and water. All qRT-PCR assays were performed in triplicate, using TaqMan ${ }^{\mathrm{TM}}$ Gene Expression 
Master Mix (Thermo Fisher Scientific, Waltham, MA, USA). Copy numbers of each target gene were calculated based on the standard curve, and HPV-16 copy numbers per cell were determined.

\subsection{Statistical Analysis}

The data were summarized by mean and standard deviation (SD) for continuous variables such as age and HPV-16 copy numbers; number of participants and percentage for the categorical variables such as gender, smoking, and race. Two-sample $t$-test or Mann-Whitney $U$ test was used for continuous variables, such as age and CD4 count.

Chi-square or Fisher's exact test was used for the categorical variables, such as gender, smoking, and race. Mann-Whitney $U$ test was used to determine whether qualitative HPV-16 detection differed between positive and negative $\mathrm{AC}$ or $\mathrm{AB}$ results. Kruskal-Wallis test was performed to assess statistical significance of HPV-16 copy numbers relative to AC or AB grade. ROC analysis was used to select the choice of a cut-off point for HPV-16 copy number to differentiate low-grade and high-grade AB. Sensitivity, specificity and Cohen's Kappa coefficient were calculated to compare the following screening assays to the gold standard of HRA with AB: AC, qualitative HPV-16 detection, and HPV-16 copy number at a cut-off of 65 copies per cell. Negative and low-grade disease grades were combined since clinical recommendations for both would typically be medical follow-up without treatment. All analyses were performed using SAS 9.4 and GraphPad Prism, and a $p$-value $<0.05$ was considered to be statistically significant.

\section{Results}

A total of 75 HIV-positive participants were enrolled in the study. Overall, 67 (89\%) were male with a mean age of 51 years. Forty-four (59\%) had an abnormal AC, of which $25(57 \%)$ had detectable HPV-16 DNA, a significantly higher rate than those with negative AC (19\%), $p=0.005$, Table 1 . The percentage of participants with detectable HPV-16 DNA did not significantly increase with disease grade severity across AC and AB grades, Figures 1 and 2. However, HPV-16 copy numbers were statistically different according to AC grade $(p=0.001)$ with the following medians (IQR): Negative 0 (0-0), ASCUS 0 (0-1.8), ASC-H 89 (0-178), LSIL 2 (0-417), and HSIL 51 (0.2-2991), Figure 3. A total of 18 participants completed an HRA with $\mathrm{AB}$ and HPV-16 copy numbers were also statistically different according to $\mathrm{AB}$ grade $(p=0.009)$ with the following medians (IQR): Negative $0(0-117.5)$, LSIL 3 (0.54-114.5) and HSIL 530 (241-18018), Figure 4.

Table 1. Demographics. Characteristics of study participants.

\begin{tabular}{ccccc}
\hline & All Participants $(\boldsymbol{n}=\mathbf{7 5})$ & Negative AC $(\boldsymbol{n}=\mathbf{3 1})$ & Positive AC $(\boldsymbol{n}=\mathbf{4 4})$ & $\boldsymbol{p}$-Value \\
\hline Age, mean (SD) & $51(10.6)$ & $51(11.9)$ & $50(9.7)$ & 0.73 \\
Gender, $n$ (\%) Male & $67(89)$ & $28(90)$ & $39(89)$ & 0.99 \\
Detectable HPV-16 & $31(41)$ & $6(19)$ & $25(57)$ & 0.005 \\
CD4 nadir count, median & 208 & 231 & 191 & 0.34 \\
\hline Smoking, $n$ (\%) & & & & \\
Current Smoker & $19(25)$ & $6(19)$ & $13(30)$ & 0.53 \\
Past Smoker & $36(48)$ & $17(55)$ & $19(43)$ & \\
Never Smoked & $20(27)$ & $8(26)$ & & \\
Race, $n$ (\%) & & & $12(27)$ & \\
Native/Alaskan American & $2(3)$ & $1(3)$ & $3(7)$ & \\
African American & $4(5)$ & $1(3)$ & $7(16)$ & \\
Asian & $11(15)$ & $4(13)$ & $22(50)$ & \\
White & $38(51)$ & $16(52)$ & $7(9)$ & \\
Hawaiian/Pacific Islander & $10(13)$ & $6(19)$ & & \\
More than One & $10(13)$ & $3(10)$ & & \\
\hline
\end{tabular}

SD: Standard Deviation; AC: Anal Cytology. 


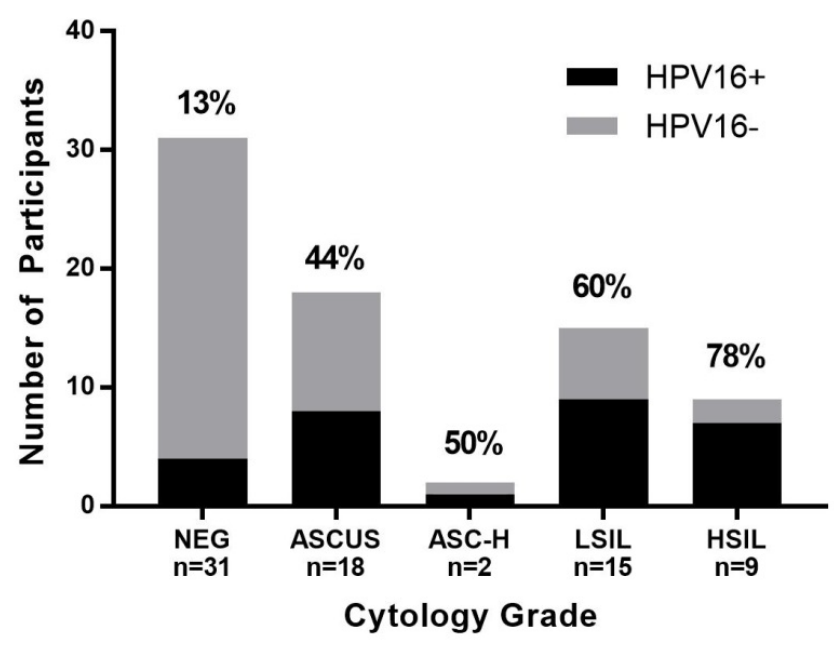

Figure 1. Detectable HPV-16 across anal cytology (AC) grades. Number of participants with positive or negative HPV-16 detection by cytology grade. Percentages depict HPV-16+.

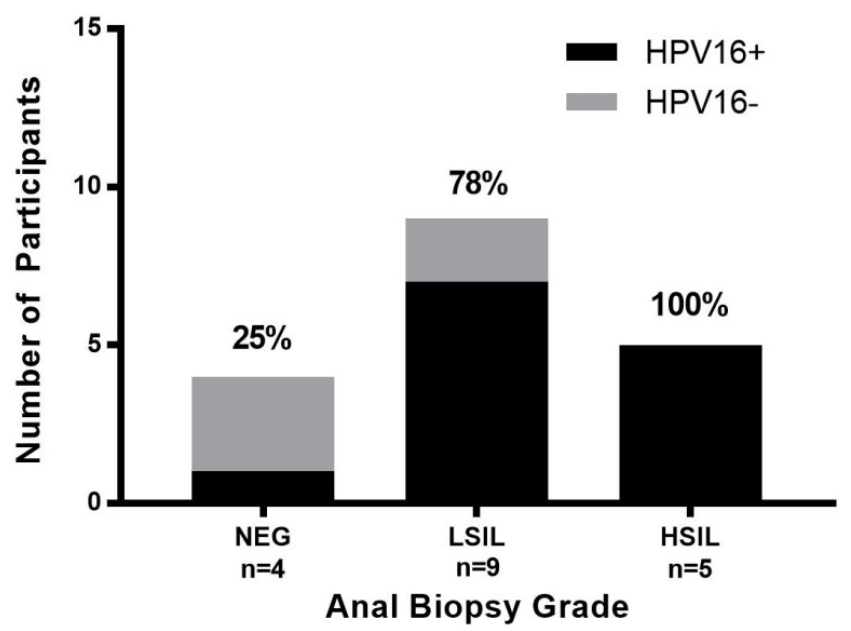

Figure 2. Detectable HPV-16 across anal biopsy (AB) grades. Number of participants with positive or negative HPV-16 detection by AB grade. Percentages depict HPV-16+.

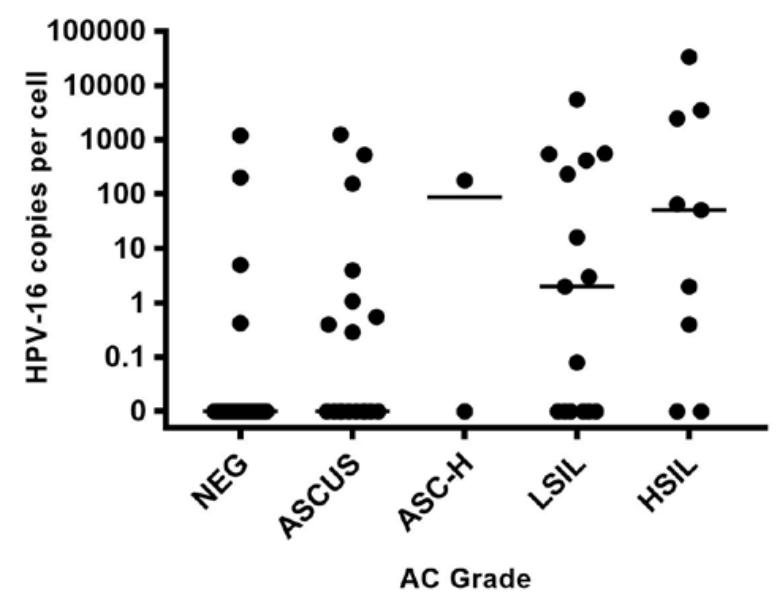

Figure 3. HPV-16 copy number by AC grade. HPV-16 copy numbers per cell of participants separated by AC grade. 


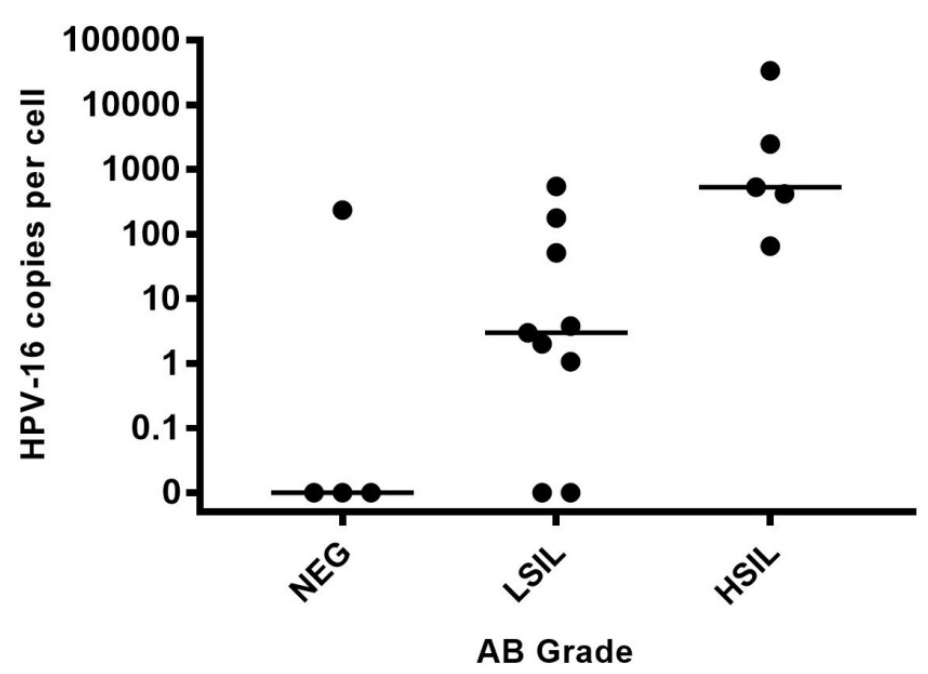

Figure 4. HPV-16 copy number by AB grade. HPV-16 copy numbers per cell of participants separated by $\mathrm{AB}$ grade.

Since higher HPV-16 copy numbers exhibited an association with more advanced disease, ROC analysis was used to determine that HPV-16 copy number $\geq 65$ copies per cell predicted HSIL with the area under the ROC curve (AUC) $=0.920$, Figure 5 .

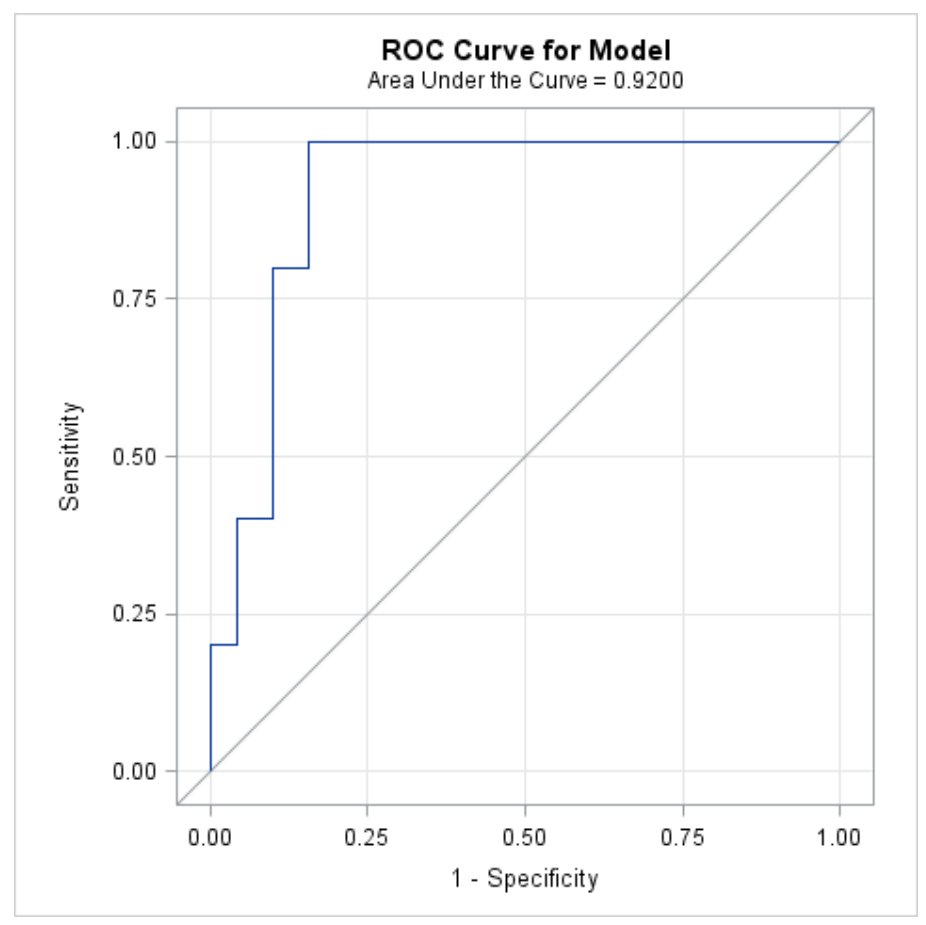

Figure 5. Receiver operating characteristic (ROC) curve used to determine high-grade squamous intraepithelial lesions (HSIL) prediction using HPV-16 copy number.

When comparing the screening assays against the current gold standard of HRA with $\mathrm{AB}$, we found that AC had lower sensitivity (0.75) with a reasonable specificity (0.86), qualitative HPV-16 had a better sensitivity (1.00) with a low specificity (0.38), and HPV-16 copy number cut-off at 65 copies per cell also had the same sensitivity as qualitative HPV-16 (1.00) but with increased specificity (0.77), Table 2. Cohen's Kappa coefficient comparing AC and AB results was 0.557 (95\% CI: 0.117, 
0.998); however, there was higher agreement between $A B$ results and HPV-16 copy number when the cut-off at 65 copies per cell was applied: 0.649 (95\% CI: 0.31, 0.989), Table 2. The Kappa coefficient comparing qualitative HPV-16 detection and AB results was 0.258 (95\% CI: 0.001, 0.515) which was lower agreement and specificity than for HPV-16 quantitation using the cut-off value of 65 copies per cell versus $A B$ results, Table 2 .

Table 2. Sensitivities, specificities and Kappa coefficients of AC, qualitative HPV-16, and HPV-16 copy $\geq 65$ vs. AB.

\begin{tabular}{cccc}
\hline & Sensitivity & Specificity & Kappa (к) (95\% CI) \\
\hline AC vs. AB & 0.75 & 0.86 & $0.557(0.117,0.998)$ \\
Qualitative HPV-16 vs. AB & 1.00 & 0.38 & $0.258(0.001,0.515)$ \\
HPV-16 copy $\geq 65$ vs. AB & 1.00 & 0.77 & $0.649(0.031,0.989)$ \\
\hline
\end{tabular}

\section{Discussion}

In our study cohort, more than half (59\%) of the participants had abnormal AC, which is consistent with other studies [14] that suggest HIV-positive individuals are at high risk for developing anal dysplasia. We also detected HPV-16 DNA in AC specimens from more than half $(57 \%)$ of the participants with abnormal AC results, a significantly higher rate $(p=0.005)$ than for those with negative AC results (19\%). Among all participants, HPV-16 was detected at a rate $(41 \%)$ consistent with other studies suggesting that HIV-infected individuals are more prone to HPV infections, which contribute to their risk for anal dysplasia/cancer [15-19]. While there was no statistical significance in qualitative HPV-16 detection across both AC and AB grades, average HPV-16 copy numbers increased at higher $\mathrm{AC}$ and $\mathrm{AB}$ grades ( $p=0.001$ and $p=0.009$ respectively). The failure to reach statistical significance for qualitative HPV-16 detection may be due to the small sample sizes that resulted after stratification into the various grades. Although AC had reasonable specificity (0.86) when compared to the current gold standard $\mathrm{AB}$, it had a decreased sensitivity (0.75) which would miss the detection of some high-grade lesions. Anal dysplasia/cancer screening guidelines have generally been adapted from cervical cancer screening because of many similarities, such as association with HPV infection. Current cervical cancer screening includes qualitative HPV detection of oncogenic types in conjunction with cytology. We found that when compared to AB, the use of qualitative HPV-16 detection had high sensitivity (1.00) but a low specificity (0.38). The use of a HPV-16 cut-off value of 65 increased the specificity (0.77) to better differentiate grade of lesions, demonstrating that quantitative HPV-16 detection using the cut-off value of 65 copies per cell may predict high-grade disease and is in better agreement with $\mathrm{AB}(0.649)$ than AC (0.557) or qualitative HPV-16 HPV detection (0.258). Genotyping studies have shown that HPV-16 DNA is frequently detected in anal specimens and is associated with anal dysplasia / cancer; however, there is limited data about variation in HPV copy numbers across $A C$ and $A B$ grades [20,21]. Our findings suggest that HPV-16 quantitation may be useful in disease classification during screening for anal dysplasia/cancer.

Limitations of this study include the small number of patients who followed up with recommended HRA and $\mathrm{AB}$ after abnormal $\mathrm{AC}$ results. The small sample size may influence the HPV-16 cut-off value. In a planned follow-up study, patients will be encouraged to return for HRA with $\mathrm{AB}$ after abnormal AC results. The study also focused on only HPV-16 even though other high-risk HPV genotypes are associated with anal dysplasia/cancer. In addition, HRA was only performed on participants who had abnormal anal cytologies. Future research could include quantitation of other HPV genotypes such as HPV-18 as well as performing HRA on all participants regardless of anal cytology results. 


\section{Conclusions}

Since HIV-positive individuals have a high risk of developing anal dysplasia/cancer they are recommended to undergo regular screening that consists of $\mathrm{AC}$ followed by an HRA with $\mathrm{AB}$ for abnormal AC. However, consistency of anal dysplasia/cancer AC screening results depends upon several factors such as sampling, operator error, and interpretation error [7]. The consequences of inconsistent $A C$ screening include cases when high-grade lesions are not found during follow-up HRA and AB, the current gold standard. In the current study, higher HPV-16 DNA copy numbers corresponded to higher $\mathrm{AC}$ and $\mathrm{AB}$ grades. We also demonstrated that a cut-off value of 65 copies per cell distinguished low-grade from high-grade disease. Using this cut-off, we found better sensitivity and agreement between HPV-16 quantitation and $A B$ than between $A C$ and $A B$, and better specificity and agreement than between qualitative HPV detection and AB. These results emphasize the impact of HPV burden on anal dysplasia disease stage and suggest that HPV-16 quantitation may improve the current screening paradigm, which may help to decrease the number of unnecessary invasive HRAs with $\mathrm{AB}$ as well as missed detection of high-grade lesions.

Author Contributions: Conceptualization, M.A.-G. and B.S.; Methodology, M.A.-G. and B.S.; Validation, M.A.-G., E.C., R.F. and B.S.; Formal Analysis, R.F. and E.L.; Investigation, M.A.-G., T.S., E.C., N.L., C.M., R.F., E.L., J.K. and B.S.; Visualization, M.A.-G., E.C., T.S.; Resources, M.A.-G. and B.S.; Data Curation, M.A.-G., T.S., E.C.; Writing-Original Draft Preparation, M.A.-G., E.C., T.S., B.S.; Writing-Review \& Editing. M.A.-G., T.S., E.C., N.L., C.M., R.F., E.L., J.K. and B.S.; Visualization-M.A.-G., B.S.; Supervision, M.A.-G., B.S.; Project Administration, M.A.-G. and B.S.; Funding Acquisition, M.A.-G., B.S.

Funding: The project described was supported by grant numbers \#U54MD007584 and \#U54MD007601 from the National Institute on Minority Health and Health Disparities (NIMHD), a component of the National Institutes of Health (NIH) and its contents are solely the responsibility of the authors and do not necessarily represent the official view of NIMHD or NIH.

Acknowledgments: Publication costs were covered by grant number \#U54MD007584 from the National Institute on Minority Health and Health Disparities (NIMHD), a component of the National Institutes of Health (NIH) and its contents are solely the responsibility of the authors and do not necessarily represent the official view of NIMHD or NIH.

Conflicts of Interest: The authors declare no conflict of interest.

\section{References}

1. Martin, F.; Bower, M. Anal intraepithelial neoplasia in HIV positive people. Sex. Transm. Infect. 2001, 77, 327-331. [CrossRef] [PubMed]

2. Burd, E.M. Human papillomavirus laboratory testing: The changing paradigm. Clin. Microbiol. Rev. 2016, 29, 291-319. [CrossRef] [PubMed]

3. Palefsky, J.M.; Holly, E.A.; Efirdc, J.T.; Da Costa, M.; Jay, N.; Berry, J.M.; Darragh, T.M. Anal intraepithelial neoplasia in the highly active antiretroviral therapy era among HIV-positive men who have sex with men. AIDS 2005, 19, 1407-1414. [CrossRef] [PubMed]

4. Chiao, E.Y.; Giordano, T.P.; Palefsky, J.M.; Tyring, S.; El Serag, H. Screening HIV-infected individuals for anal cancer precursor lesions: A systematic review. Clin. Infect. Dis. 2006, 43, 223-233. [CrossRef] [PubMed]

5. Carvalho, N.S.; Ferreira, A.M.; Bueno, C.C. HPV infection and intraepithelial lesions from the anal region: How to diagnose? Braz. J. Infect. Dis. 2011, 15, 473-477. [CrossRef] [PubMed]

6. Darragh, T.M.; Colgan, T.J.; Cox, J.T.; Heller, D.S.; Henry, M.R.; Luff, R.D.; McCalmont, T.; Nayar, R.; Palefsky, J.M.; Stoler, M.H.; et al. The lower anogenital squamous terminology standardization project for HPV-associated lesions: Background and consensus recommendations from the college of american pathologists and the american society for colposcopy and cervical pathology. Arch. Pathol. Lab. Med. 2012, 136, 1266-1297. [CrossRef] [PubMed]

7. Cachay, E.R.; Mathews, W.C. Human papillomavirus, anal cancer, and screening considerations among HIV-infected individuals. AIDS Rev. 2013, 15, 122-133. [PubMed]

8. Mathews, W.C.; Cachay, E.R.; Caperna, J.; Sitapati, A.; Cosman, B.; Abramson, I. Estimating the accuracy of anal cytology in the presence of an imperfect reference standard. PLoS ONE 2010, 5, e12284. [CrossRef] [PubMed] 
9. Baena, A.; Guevara, E.; Almonte, M.; Arias-Stella, J.; Sasieni, P.; Sanchez, G.I. Factors related to inter-observer reproducibility of conventional pap smear cytology: A multilevel analysis of smear and laboratory characteristics. Cytopathology 2017, 28, 192-202. [CrossRef] [PubMed]

10. Stoler, M.H.; Schiffman, M.; Atypical Squamous Cells of Undetermined Significance-Low-Grade Squamous Intraepithelial Lesion Triage Study Group. Interobserver reproducibility of cervical cytologic and histologic interpretations: Realistic estimates from the ascus-lsil triage study. JAMA 2001, 285, 1500-1505. [CrossRef] [PubMed]

11. Gonzalez-Hernandez, L.A.; Flores-Miramontes, M.G.; Aguilar-Lemarroy, A.; Quintanilla-Pena, K.S.; Martin-Amaya-Barajas, F.L.; Ramos-Solano, M.; Enciso Gomez, L.F.; Andrade-Villanueva, J.F.; Jave-Suarez, L.F. HPV genotypes detected by linear array and next-generation sequencing in anal samples from HIV positive men who have sex with men in mexico. Arch. Virol. 2018, 163, 925-935. [CrossRef] [PubMed]

12. Dona, M.G.; Benevolo, M.; Latini, A.; Rollo, F.; Colafigli, M.; Frasca, M.; Zaccarelli, M.; Giglio, A.; Moretto, D.; Pescarmona, E.; et al. Anal cytological lesions and HPV infection in individuals at increased risk for anal cancer. Cancer Cytopathol. 2018, 126, 461-470. [CrossRef] [PubMed]

13. Camargo, M.; Soto-De Leon, S.; Sanchez, R.; Munoz, M.; Vega, E.; Beltran, M.; Perez-Prados, A.; Patarroyo, M.E.; Patarroyo, M.A. Detection by pcr of human papillomavirus in colombia: Comparison of gp5+/6+ and my09/11 primer sets. J. Virol. Methods 2011, 178, 68-74. [CrossRef] [PubMed]

14. Gaisa, M.; Sigel, K.; Hand, J.; Goldstone, S. High rates of anal dysplasia in HIV-infected men who have sex with men, women, and heterosexual men. AIDS 2014, 28, 215-222. [CrossRef] [PubMed]

15. Hernandez, A.L.; Efird, J.T.; Holly, E.A.; Berry, J.M.; Jay, N.; Palefsky, J.M. Risk factors for anal human papillomavirus infection type 16 among HIV-positive men who have sex with men in san francisco. J. Acquir. Immune Defic. Syndr. 2013, 63, 532-539. [CrossRef] [PubMed]

16. Utaipat, U.; Siriaunkgul, S.; Supindham, T.; Saokhieo, P.; Chaidaeng, B.; Wongthanee, A.; Settakorn, J.; Sukpan, K.; Ruanpeng, D.; Kosashunhanan, N.; et al. Association of cytologic grade of anal "pap" smears with viral loads of human papillomavirus types 16, 18, and 52 detected in the same specimens from men who have sex with men. J. Clin. Virol. 2016, 85, 48-55. [CrossRef] [PubMed]

17. Poizot-Martin, I.; Henry, M.; Benhaim, S.; Obry-Roguet, V.; Figarella, D.; Tamalet, C. High level of HPV 16 and 18 DNA load in anal swabs from male and female HIV-1 infected patients. J. Clin. Virol. 2009, 44, 314-317. [CrossRef] [PubMed]

18. Van Aar, F.; Mooij, S.H.; van der Sande, M.A.; Speksnijder, A.G.; Stolte, I.G.; Meijer, C.J.; Verhagen, D.W.; King, A.J.; de Vries, H.J.; Schim van der Loeff, M.F. Anal and penile high-risk human papillomavirus prevalence in HIV-negative and HIV-infected msm. AIDS 2013, 27, 2921-2931. [CrossRef] [PubMed]

19. Palefsky, J.M.; Holly, E.A.; Ralston, M.L.; Jay, N. Prevalence and risk factors for human papillomavirus infection of the anal canal in human immunodeficiency virus (HIV)-positive and HIV-negative homosexual men. J. Infect. Dis. 1998, 177, 361-367. [CrossRef] [PubMed]

20. Wieland, U.; Hellmich, M.; Wetendorf, J.; Potthoff, A.; Hofler, D.; Swoboda, J.; Fuchs, W.; Brockmeyer, N.; Pfister, H.; Kreuter, A. Smoking and anal high-risk human papillomavirus DNA loads in HIV-positive men who have sex with men. Int. J. Med. Microbiol. 2015, 305, 689-696. [CrossRef] [PubMed]

21. Tamalet, C.; Obry-Roguet, V.; Ressiot, E.; Bregigeon, S.; Del Grande, J.; Poizot-Martin, I. Distribution of human papillomavirus genotypes, assessment of HPV 16 and 18 viral load and anal related lesions in HIV positive patients: A cross-sectional analysis. J. Med. Virol. 2014, 86, 419-425. [CrossRef] [PubMed]

(C) 2018 by the authors. Licensee MDPI, Basel, Switzerland. This article is an open access article distributed under the terms and conditions of the Creative Commons Attribution (CC BY) license (http://creativecommons.org/licenses/by/4.0/). 